\title{
Behaviour of time-dependent materials exposed to periodical loading
}

\author{
B. Zupancic ${ }^{1, a}$, and I. Emri ${ }^{1}$
}

${ }^{1}$ Center for Experimental Mechanics, Faculty of Mechanical Engineering, University of Ljubljana, Askerceva 6, 1000 Ljubljana, Slovenia

\begin{abstract}
Paper presents methodology for analyzing behaviour of time-dependent (viscoelastic) materials when exposed to periodical (cyclic) loading. Within each loading cycle time-dependent material undergoes a combination of the creep and retardation process. At certain conditions the retardation process between two loading cycles cannot be fully completed to a strain free state. Consequently, strain starts to accumulate, which leads to hardening of the material and ultimately to the failure of polymeric product. Critical frequency of the applied periodical loading depends on the material retardation time (location of mechanical spectrum), while the magnitude of accumulated strain on the strength of corresponding discrete spectrum lines. The shape of mechanical spectrum defines the intensity and the magnitude of accumulated strain. Thus, the mechanical spectrum of time-dependent material is the most important function for predicting durability of dynamically loaded polymeric products. In continuation we present mathematical methodology for predicting durability of periodically loaded polymeric components of drive belt. Methodology includes numerical calculations of accumulated strain as a function of the number of loading cycles and function of loading angular velocity. Based on these calculations we can determine the critical angular velocity area of periodical loading where the strain accumulation is the most intensive.
\end{abstract}

\section{Introduction}

Loading conditions of the drive belt were modelled based on the results that were obtained by the FEM analysis of the stress-strain state of the belt within a complete loading cycle [1]. Schematics of the belt loading conditions and belt/pulley geometry is shown in Figure 1.

a e-mail : barbara.zupancic@fs.uni-1j.si

This is an Open Access article distributed under the terms of the Creative Commons Attribution-Noncommercial License 3.0, which permits unrestricted use, distribution, and reproduction in any noncommercial medium, provided the original work is properly cited. 


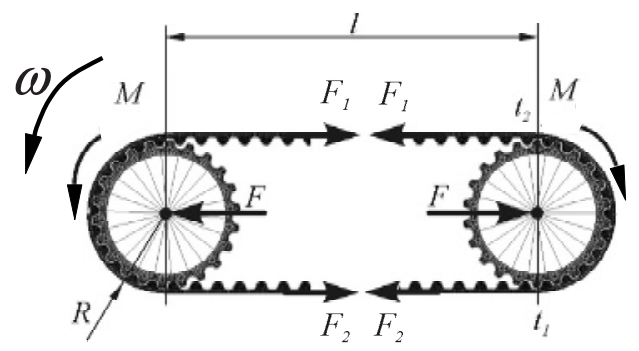

Fig. 1. Schematics of the belt loading conditions and belt/pulley geometry [1].

For the selected location on the tooth of the belt the FEM analysis has shown that the evolution of the shear stress within one loading cycle may be modelled as a tooth-like function [1], shown schematically in Figure 2.

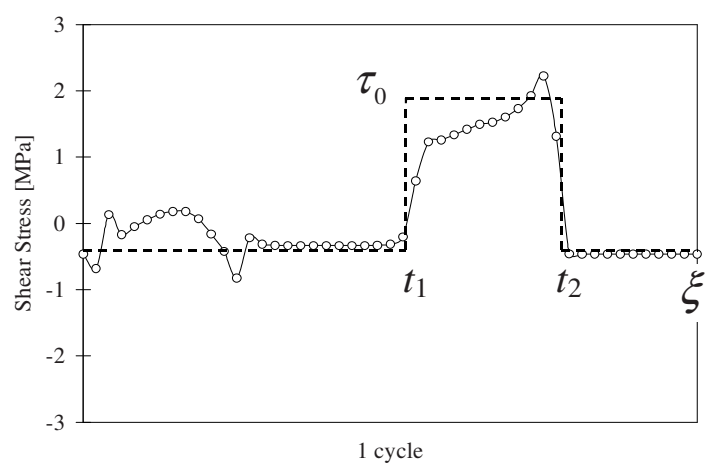

Fig. 2. Evolution of the shear stress for a selected point on the tooth of the belt within one loading cycle and modelling of the shear stress within one loading cycle with a tooth-like function [1].

Time $t_{1}$ denotes the beginning of the loading phase, $t_{2}$ denotes the end of the loading phase within one loading cycle, while $\xi$ the duration of one loading cycle (Figure 2). $\tau_{0}$ in Figure 2 presents the magnitude of the loading shear stress. Modelled loading conditions in terms of the shear stress may be mathematically described as [1],

$$
\tau(t)=\tau(0) h(t)+\tau_{0} \sum_{n=1}^{n=N}\left\{h\left[t-t_{1}-(n-1) \xi\right]-h\left[t-t_{2}-(n-1) \xi\right]\right\} .
$$

Times $t_{1}, t_{2}$, and the duration of one load cycle $\xi$ are functions of geometry of the belt drive, and angular velocity of the pulleys, $\omega$. Thus, $t_{1}=(l+\pi R) /(\omega R), t_{2}=(l+2 \pi R) /(\omega R)$, and $\xi=(2 l+2 \pi R) /(\omega R)$, where $l$ is the distance between the axes of the two pulleys, $R$ is the radius of the pulleys, and $N$ is the number of cycles to which the belt has been exposed. 


\section{Modelling of strain accumulation process}

The strain response of the viscoelastic material exposed to the shear stress loading may be expressed as [2],

$$
\gamma(t)=\int_{0}^{t} J(t-s) \frac{\partial \tau(s)}{\partial s} d s
$$

Creep compliance material function $J(t)$ from Eq. (2) may be expressed in terms of discrete retardation spectrum as,

$$
J(t)=J_{g}+\sum_{i=1}^{K} L_{i}\left(1-e^{-\frac{t}{\lambda_{i}}}\right)
$$

Here $J_{g}$ denotes the glassy compliance, $\lambda_{i}$ the retardation times where the corresponding spectrum lines $L_{i}=L\left(\lambda_{i}\right)$ are located, and $K$ represents the number of spectral lines required to model a selected material.

Introducing Eq. (1) and Eq. (3) into the Eq. (2), yields the following expression for the cumulative accumulated strain [1],

$$
\Gamma_{N}(t)=\tau_{0} \sum_{n=1}^{n=N} \sum_{i=1}^{K} L_{i}\left[\exp \left(-\frac{t}{\lambda_{i}}+\frac{2 n(l+\pi R)-l}{\omega R \lambda_{i}}\right)-\exp \left(-\frac{t}{\lambda_{i}}+(2 n-1) \frac{l+\pi R}{\omega R \lambda_{i}}\right)\right]
$$

Eq.4 for accumulated strain at the end of $N$ completed cycles, i.e., at $t=t_{N}$, expressed as $t_{N}=N \xi=2 N \frac{l+\pi R}{\omega R}=2 N \frac{\kappa+\pi}{\omega}$ where $\kappa=l / R$, becomes

$$
\Gamma_{N}(N)=\tau_{0} \sum_{n=1}^{n=N} \sum_{i=1}^{K} L_{i}\left[1-\exp \left(-\frac{\pi}{\omega \lambda_{i}}\right)\right] \exp \left(-\frac{(\kappa+\pi)(2(N-n)+1)-\pi}{\omega \lambda_{i}}\right) .
$$

Eq. (5) describes the time-dependent evolution of the strain accumulation in the material as a function of time-dependent material property, as a function of the number of loading cycles, $N$, to which the belt has been exposed to, as a function of the angular velocity, $\omega$, and as a function of geometry parameter $\kappa=\frac{l}{R}$, where $l$ is the distance between the axes of the two pulleys, and $R$ is the radius of the pulleys of the belt drive, [1]. 


\section{Strain accumulation analysis}

To analyze the effect of different time-dependent material properties on the evolution of the accumulated strain we selected the envelopes of the five retardation spectra that are shown in Figure 3. Box, Lorentzian, and Gauss, retardation spectra are chosen to be symmetrical in respect to $\log \lambda=0$, while the ramp spectrum, is asymmetric and it becomes constant at point $\log \lambda=0$, whereas the wedge spectrum, monotonically decreases in respect to $\log \lambda=0$.

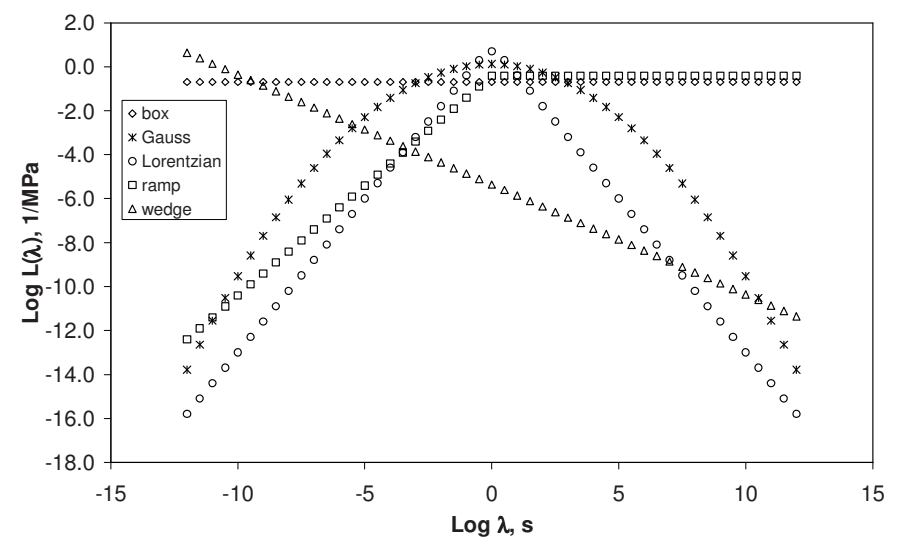

Fig. 3. Envelopes of the five different spectra: box, Gauss, Lorentzian, ramp, wedge.

Using the five spectra we can now generate the corresponding response creep compliance functions, $J(t)$, using Eq. (3). The response functions corresponding to the five spectra are shown in Figure 4.

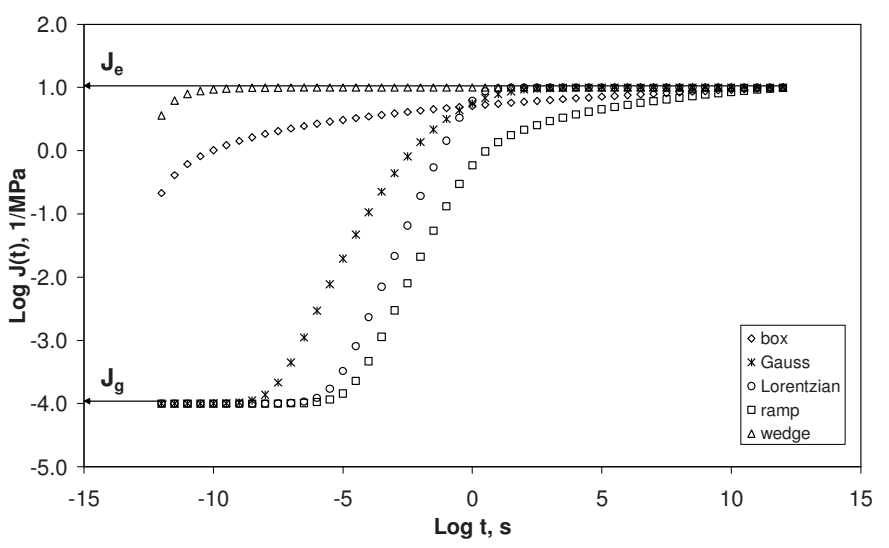

Fig. 4. Creep compliance functions, $J(t)$, corresponding to the five retardation spectra.

In this case we have chosen different shapes of the retardation spectra, $L(\lambda)=\left\{L_{i}, \lambda_{i} ; i=1,2, \cdots, K\right\}$, and then calculate the corresponding response functions, $J(t)$. In reality the situation is just opposite. The retardation spectrum can not be measured directly. We can measure the response functions in time, $J(t)$, and the corresponding retardation spectrum is then calculated by solving the inverse problem of the Eq. Error! Reference source not found., using one of the existing algorithms, e.g., the Windowing Algorithm [3]. 


\subsection{The effect of loading cycle number on the strain accumulation}

For selected five types of retardation spectra we firstly analyzed the the effect of the number of loading cycles on the strain accumulation process by generating $3 \mathrm{D}$ diagrams of cumulative accumulated strain, $\Gamma(N, \omega, \kappa=\pi)$ as function of the number of loading cycles, $N$, and angular velocity, $\omega$, for fixed value of geometry parameter, $\kappa=l / R=\pi$. Such diagram is demonstrated in Figure 5 for the Gauss spectrum distribution. Black contour denotes cumulative accumulated strain as a function of the number of loading cycles, $N$ at angular velocity $\omega=100 / \mathrm{sec}$, and given geometry, $\kappa=\pi$.

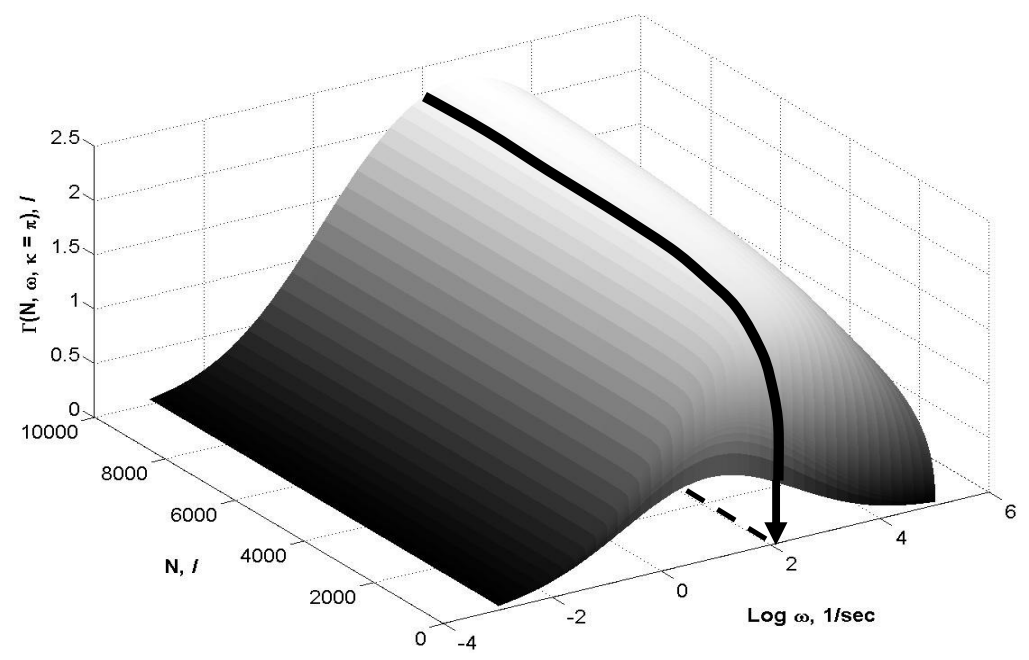

Fig. 5. Diagram of cumulative accumulated strain, $\Gamma(N, \omega, \kappa=\pi)$, as function of the number of loading cycles, $N$, and angular velocity, $\omega$, for Gauss spectrum distribution, where black contour denotes cumulative accumulated strain as a function of the number of loading cycles, $N$ at angular velocity $\omega=100 / \mathrm{sec}$, and given geometry, $\kappa=\pi$.

Let us now analyze the cumulative accumulated strain as a function of the number of loading cycles, for angular velocity, $\omega=100 / \mathrm{sec}$, and $\kappa=\pi$ for all five spectra. Diagram of cumulative accumulated strain as function of the number of loading cycles, $N$ for all five spectra is displayed in Figure 6.

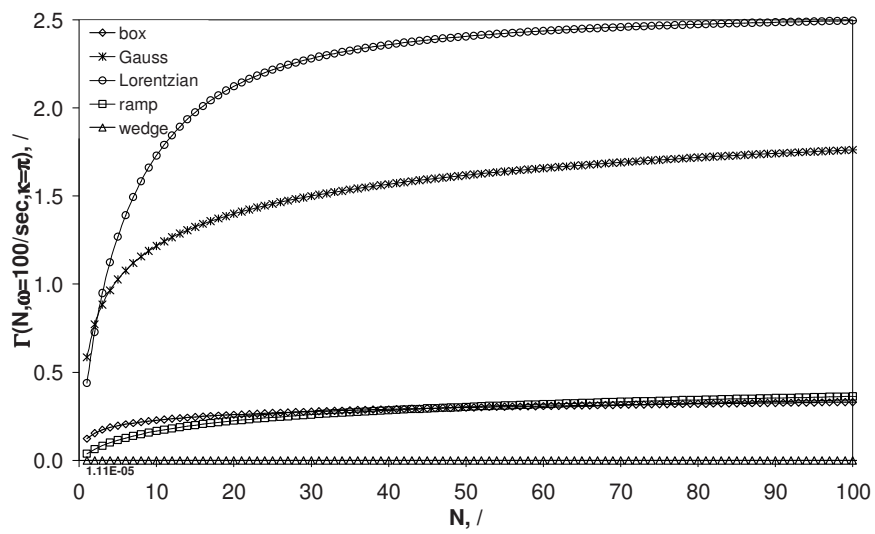

Fig. 6. Diagram of cumulative accumulated strain, $\Gamma(N, \omega=100 / \mathrm{sec}, \kappa=\pi)$ as function of the number of loading cycles, $N$, for five different material spectra. 
For better visualization of results, the diagrams are presented only within the scale of 100 loading cycles, despite the fact that the calculations were done for 10000 cycles.

The most intensive strain accumulation for $\kappa=\pi$ and $\omega=100 / \mathrm{sec}$ exhibits material with the Lorentzian retardation spectrum (Figure 6). The next conclusion can also be made: The strain accumulation process is the most intensive at the beginning of the drive belt operation, and diminishes with the number of loading cycles which can be observed as a slower increasing tendency of cumulative accumulated strain after certain number of loading cycles (see Figure 6).

Since we expect for the operating conditions to also have important effect on the strain accumulation process, we analyze this effect in continuation.

\subsection{The effect of the operating angular velocity on the strain}

The information on critical operating conditions where, for a given material, the strain accumulation is the most intensive is very important, since operating under such conditions may lead to a failure of the drive belt.

To analyze how cumulative accumulated strain depends on the distribution of retardation spectra for $\omega$ we followed the results from $3 \mathrm{D}$ diagrams of cumulative accumulated strain, $\Gamma(N, \omega, \kappa=\pi)$ as function of the number of loading cycles, $N$, and angular velocity, $\omega$, for fixed value of geometry parameter, $\kappa=l / R=\pi$ (Figure 5). From the same diagram that is presented in Figure 5 for the Gauss spectrum distribution we can analyze the effect of operating angular velocity by fixing the number of loading cycles to $N=10000$. Fixation of parameter is demonstrated in Figure 7 with the black contour that denotes cumulative accumulated strain as a function of angular velocity, $\omega$, for fixed number of loading cycles, $N=10000$, and given geometry, $\kappa=\pi$.

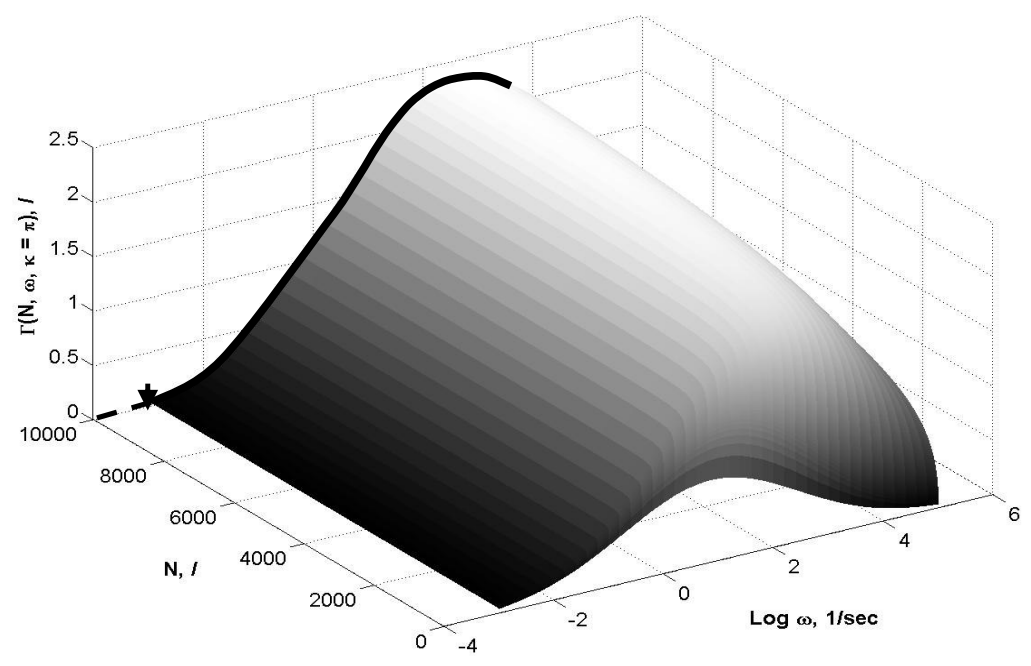

Fig. 7. Diagram of cumulative accumulated strain, $\Gamma(N, \omega, \kappa=\pi)$, as function of the number of loading cycles, $N$, and angular velocity, $\omega$, for Gauss spectrum distribution, where black contour denotes cumulative accumulated strain as a function of angular velocity, $\omega$, for fixed number of loading cycles, $N=10000$, and given geometry, $\kappa=\pi$.

Let us follow the procedure above and analyze the cumulative accumulated strain as a function of operating angular velocity, $\omega$, for $N=10000$ and $\kappa=\pi$ for all five spectra. The diagrams of cumulative accumulated strain $\Gamma(\omega, \kappa=\pi, N=10000)$ as a function of operating angular velocity, $\omega$ for all five spectra are displayed in Figure 8. 


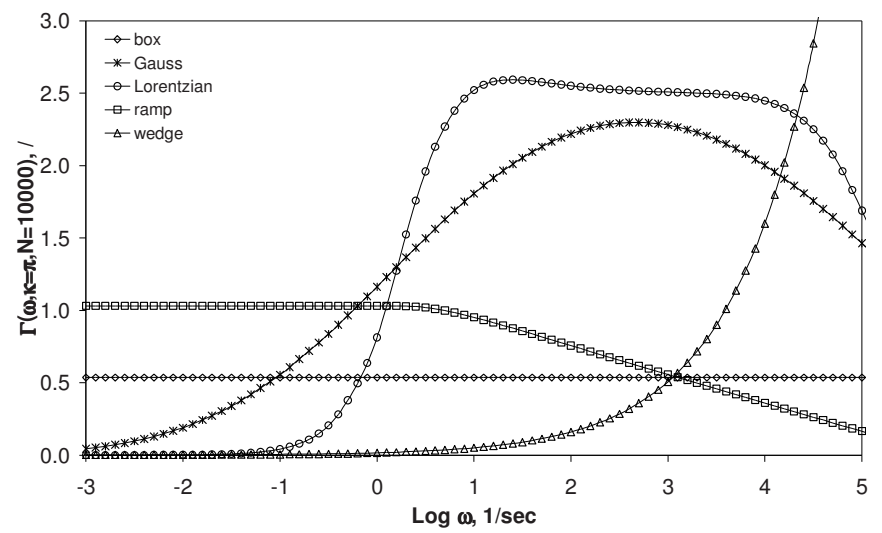

Fig. 8. Diagram of cumulative accumulated strain, $\Gamma(\omega, \kappa=\pi, N=10000)$, as function of operating angular velocity, $\omega$, for five different material spectra.

From the observation of diagrams in Figure 8 we may conclude the following:

In the case of the box distribution we can observe that the cumulative accumulated strain is essentially independent of the angular velocity at which the drive belt operates since the magnitude of accumulated strain is the same in the whole range of operating angular velocity.

For the wedge retardation spectrum we observe that strain accumulation process much less intensive in comparison to other spectra.

For the ramp spectrum we may observe that the strain accumulation is more intensive at lower angular velocities, which corresponds the fact that spectrum lines at high response times are responsible for the strain accumulation at low drive-belt angular velocities (high frequencies), and vice versa.

The strain accumulation process is the most intensive for the two spectra, i.e., for the Gauss and the Lorentzian spectrum, that are the spectra with more noticeable peak in spectrum distribution.

From this we may conclude, that material with narrower peak in spectrum distribution will exhibit the most intensive strain accumulation, considering different operating conditions, in the narrower angular frequency domain, while for material with broader spectrum distribution (as for example box spectrum) broader operating angular velocity area will cause the most intensive strain accumulation. Besides that, we can expect higher magnitude of the accumulated strain for material with narrower spectrum peak in comparison to broader spectrum.

We may then define the critical angular velocity area, where the strain accumulation process is the most intensive. Based on that we can obtain the information on durability of a belt made from a selected material by suggesting the criterion that is presented in continuation.

\subsection{Criterion for determination of critical angular velocity}

We demonstrate the establishment of criterion to determine critical angular velocity area, where the strain accumulation is the most intensive, again for the case of the Gauss spectrum. Starting from the diagram of cumulative accumulated strain, $\Gamma(N, \omega, \kappa=\pi)$ as function of the number of loading cycles, $N$, and angular velocity, $\omega$, we define different upper limits of the accumulated strain, as shown with the cross-section plane (solid black contour) in Figure 9, and project the cross-section curve (dashed black line) onto $n-\omega$ plane, we obtain different levels of critical angular velocity areas. Within the "critical area" we may expect the most intensive strain accumulation. 


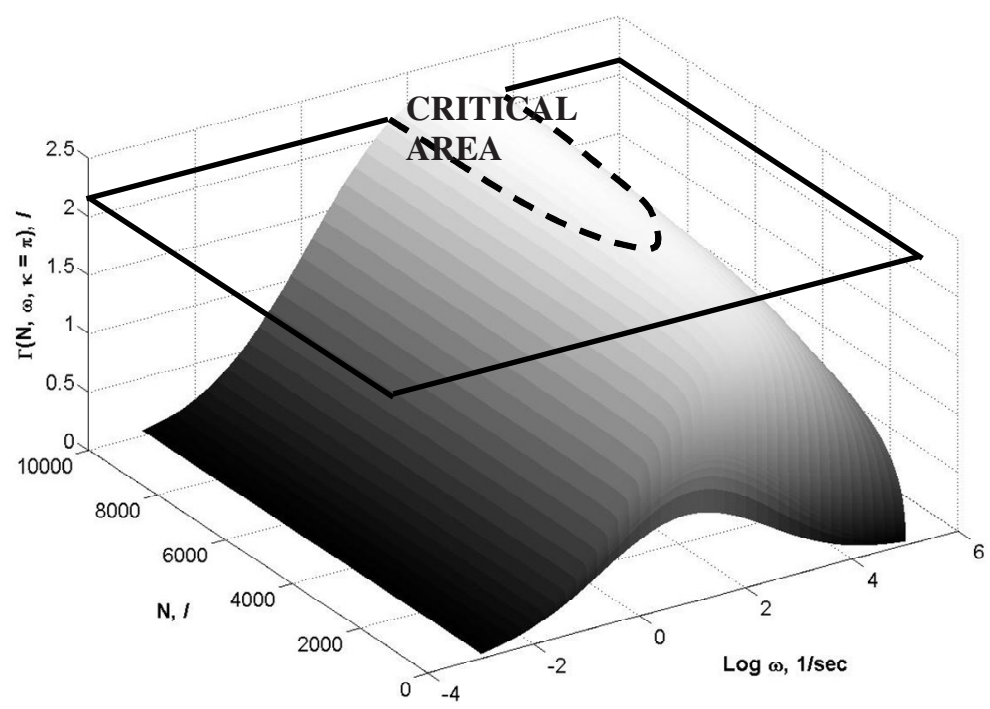

Fig. 9. 3D diagram of accumulated strain, $\Gamma(N, \omega, \kappa=\pi)$, as a function loading cycles, $N$, and angular velocity, $\omega$.

\section{Conclusions}

We developed the theoretical approach for modelling the strain accumulation process in periodically loaded polymeric products. The strain accumulation process was analyzed for materials with different spectrum distributions, and demonstrated on the Gauss spectrum. The strain accumulation process was analyzed as function of the loading angular velocity and the number of loading cycles. Based on these analyses and established criterion for determination of critical angular velocity we propose the methodology for predicting the durability (the life span) of periodically loaded polymeric products by following the procedure described above.

\section{References}

1. Emri, I., Kramar, J., Hribar, A., Nikonov, A., Florjančič, U., Mechanics of Time-Dependent Materials, 10(3), 245-262 (2006)

2. Tschoegl, N.W., The Phenomenological Theory of Linear Viscoelastic Behavior: An Introduction (1989)

3. Emri, I., Tschoegl, N. W.: Generating line spectra from experimental responses. Part I: Relaxation modulus and creep compliance. Rheologica Acta 32, 311-321 (1993) 